\title{
Current Challenges for IDO2 as Target in Cancer Immunotherapy
}

\author{
Giada Mondanelli ${ }^{1}$, Martina Mandarano ${ }^{2}$, Maria Laura Belladonna ${ }^{1}$, Chiara Suvieri ${ }^{1}$, \\ Cristina Pelliccia ${ }^{2}$, Guido Bellezza ${ }^{2}$, Angelo Sidoni ${ }^{2}$, Agostinho Carvalho ${ }^{3,4 \dagger}$, \\ Ursula Grohmann ${ }^{1 * t}$ and Claudia Volpi ${ }^{1 * t}$
}

${ }^{1}$ Department of Medicine and Surgery, Section of Pharmacology, University of Perugia, Perugia, Italy, ${ }^{2}$ Department of Medicine and Surgery, Section of Anatomic Pathology and Histology, University of Perugia, Perugia, Italy, ${ }^{3}$ Life and Health Sciences Research Institute (ICVS), School of Medicine, University of Minho, Braga, Portugal, ${ }^{4}$ ICVS/3B's - PT Government Associate Laboratory, Braga/Guimarães, Portugal

\section{OPEN ACCESS}

Edited by: Fernando Guimaraes, University of Queensland, Australia

Reviewed by: Stefano Ugel, University of Verona, Italy Alexander Steinkasserer, University Hospital Erlangen, Germany

*Correspondence: Claudia Volpi claudia.volpi@unipg.it Ursula Grohmann ursula.grohmann@unipg.it

${ }^{t}$ These authors share senior authorship

Specialty section: This article was submitted to Cancer Immunity and Immunotherapy, a section of the journal Frontiers in Immunology

Received: 12 March 2021 Accepted: 01 April 2021 Published: 21 April 2021

Citation:

Mondanelli G, Mandarano $M_{\text {, }}$ Belladonna ML, Suvieri C, Pelliccia C, Bellezza G, Sidoni A, Carvalho A, Grohmann U and Volpi C (2021) Current Challenges for IDO2 as Target in Cancer Immunotherapy.

Front. Immunol. 12:679953. doi: 10.3389/fimmu.2021.679953
Immune checkpoint inhibitors have revolutionized the clinical approach of untreatable tumors and brought a breath of fresh air in cancer immunotherapy. However, the therapeutic effects of these drugs only cover a minority of patients and alternative immunotherapeutic targets are required. Metabolism of L-tryptophan (Trp) via the kynurenine pathway represents an important immune checkpoint mechanism that controls adaptive immunity and dampens exaggerated inflammation. Indoleamine 2,3dioxygenase 1 (IDO1), the enzyme catalyzing the first, rate-limiting step of the pathway, is expressed in several human tumors and IDO1 catalytic inhibitors have reached phase III clinical trials, unfortunately with disappointing results. Although much less studied, the IDO1 paralog IDO2 may represent a valid alternative as drug target in cancer immunotherapy. Accumulating evidence indicates that IDO2 is much less effective than IDO1 in metabolizing Trp and its functions are rather the consequence of interaction with other, still undefined proteins that may vary in distinct inflammatory and neoplastic contexts. As a matter of fact, the expression of IDO2 gene variants is protective in PDAC but increases the risk of developing tumor in NSCLC patients. Therefore, the definition of the IDO2 interactome and function in distinct neoplasia may open innovative avenues of therapeutic interventions.

Keywords: IDO2, tryptophan metabolism, pseudoenzymes, NSCLC, PDAC

\section{INTRODUCTION}

Over the course of evolution, the metabolism of L-tryptophan (Trp), an essential amino acid for mammals, has evolved to be a primary control node in the regulation of immune responses (1). In this regard, the most important enzyme is indoleamine 2,3-dioxygenase 1 (IDO1), a monomeric, heme-containing enzyme that catalyzes the initial, rate-limiting step in the degradation of Trp along the so-called kynurenine pathway $(2,3)$. L-kynurenine $(\mathrm{Kyn})$, the first product of this pathway, promotes immunoregulatory effects via activation of the aryl hydrocarbon receptor (AhR) in dendritic cells (DCs) and T lymphocytes (4-7). By degrading Trp, IDO1 also depletes the essential amino acid in microenvironments, thus activating the general control non-depressible 2 (GCN2) kinase pathway and the dysfunction of $\mathrm{T}$ cells $(8,9)$. In addition to catalytic activity, IDO1 is 
endowed with a signaling function that, upon tyrosine phosphorylation of immune tyrosine inhibitory motifs (ITIMs) in the small noncatalytic domain of the enzyme, allows the direct interaction with Src homology region 2 domain-containing phosphatase-1 and -2 (SHP-1 and SHP-2, respectively) and confers long-term immunoregulatory properties on DCs (10, 11). The same domain also contains a YxxM motif that, once tyrosine phosphorylated, binds the p85 subunit of class I phosphoinositide 3-kinases (PI3Ks) that drive IDO1 trafficking from cytosol (where exerts the catalytic function) to early endosomes, thus favoring IDO1 signaling activity (12).

IDO1 is expressed in several human tumors and immune cells infiltrating the tumor mass (13) and, for this reason, IDO1 catalytic inhibitors have been used as experimental drugs in cancer immunotherapy $(9,14)$. One of these inhibitors, epacadostat, was recently coadministered with pembrolizumab, an immune checkpoint inhibitor, in patients with unresectable or mestastatic melanoma in a phase III trial. However, the results were disappointing, as epacadostat did not show the efficacy observed in the previous phase II trial (15). Several causes may have determined this failure, including an inadequate selection of patients. However, considering its complex functional dynamics as described above, IDO1 may represent a hard molecule to be exploited as an effective drug target.

Some years ago, a paralog of IDO1, i.e., IDO2, was discovered. In accordance with those studies, the IDO1 gene (expressed in mammals and fungi) derived from the duplication of IDO2 (expressed in all organisms, including bacteria), thus considered more ancestral than IDO1, and the two genes can be detected in tandem in chromosome 8 in both humans and rodents (16). Although it can also initiate the kynurenine pathway, IDO2 affinity for the Trp substrate and catalytic efficacy in producing Kyn are very low or almost negligible (17). Therefore, IDO2 may contribute only a minimal role to overall Trp metabolism (18). Nevertheless, IDO2 is expressed at high levels in some human tumors and, therefore, understanding its true function/s in neoplastic contexts may propel the development of new drugs targeting enzymes of the kynurenine pathway in cancer immunotherapy.

\section{THE "MYSTERY" OF IDO2 FUNCTION: HINTS FROM AUTOIMMUNE/CHRONIC INFLAMMATORY DISEASES}

Since the discovery of IDO2, the primary efforts of the scientific community were addressed to deciphering its role in the modulation of immune responses, assuming that a remarkable sequence homology with IDO1 was accompanied by a parallel analogy in the immunoregulatory functions. A first attempt to discern the physiological and pathophysiological functions of IDO2 was made through the generation of mice deficient in the Ido2 gene. The characterization of these genetically deficient mice highlighted that IDO1 and IDO2 show some important differences. As a matter of fact, the ablation of IDO2 did not affect Kyn circulating levels, suggesting a specific role for IDO2 and distinct from the enzymatic function. IDO2 was essential for IDO1-dependent induction of $\mathrm{T}$ regulatory (Treg) cells and, in IDO1 knockout mice, a great amount of Ido2 transcripts were subjected to alternative splicing, implying a mutual influence between the two paralogues regarding their expression and function (19). Nevertheless, in a classical model of hapteninduced contact hypersensitivity (CHS), the contribution of IDO2 in the adaptive inflammatory response was remarkably different from that of IDO1, with a reduced response and a significant impairment in proinflammatory cytokines production in IDO2-deficient mice (19).

The positive role of IDO2 in the development of inflammatory processes was further and elegantly demonstrated by means of a murine model of autoimmune arthritis, i.e., the KRN.g7 mice, genetically deficient for either the Ido1 or Ido2 gene, which revealed that IDO2, but not IDO1, is necessary for arthritis development. IDO2-deficient mice showed a delayed onset and reduced arthritis severity, due to a reduction in pathogenic antibody-secreting cells and corresponding decrease in autoantibodies (20). A thorough analysis of the IDO2 involvement in the pathogenesis of arthritis revealed that IDO2 participates in the initiation stage of the response prior to the generation of autoantibodies; however, no clues for the exact mechanism of action of IDO2 could be obtained. In the same experimental setting, the specific silencing of IDO2 in B cells significantly reduced total arthritis severity, confirming the role of IDO2 in disease initiation and progression and pinpointing IDO2 as an innovative target for the treatment of this autoimmune disease (21). Again, no evidence emerged from these studies revealing the molecular mechanism of IDO2 in regulating the autoimmune response in the arthritis model. In line with these observations, in humans, the expression of an IDO2 variant lacking catalytic activity is associated with reduced risk of Crohn's disease (7).

In contrast with these studies, in a model of psoriasis-like inflammation, the manifestations of the disease were significantly worse in the IDO2 $\mathrm{KO}$ mice (22). In fact, full active IDO2 was endowed with the ability to control the production of proinflammatory IL-17, thus contributing to the suppression of skin inflammation. Therefore, the results obtained in the murine model of psoriasis add more complexity than reinforcing the hypothesis of a proinflammatory role of IDO2.

A perspective that could reconcile the apparent divergence of the results obtained in different experimental models of inflammation/autoimmunity is that the activity of IDO2 may be strictly related to the physiopathologic context and cellular microenvironment. In support of this hypothesis, a recent study revealed that, in two different cohorts of patients with aspergillosis, specific and different patterns of IDO2 single nucleotide polymorphisms (SNPs) can be observed. More specifically, in patients with cystic fibrosis, SNPs that profoundly affect IDO2 expression and/or function did not associate with an increased risk of aspergillosis, whereas the same SNPs were required for optimal antifungal activity in patients who have undergone hematopoietic stem cell transplantation (23). 


\section{IDO2 EXPRESSION AND FUNCTION IN TUMORS: A MATTER OF GENETICS}

In general, overexpression of IDO2 in tumors appears to be less frequent than IDO1 (13). More recently, human gastric, colorectal, and renal carcinomas have been found to constitutively express both IDO1 and IDO2 (24), and the same has been observed in brain tumors (25). Interestingly, IDO2 is particularly overexpressed in pancreatic ductal adenocarcinoma (PDAC) (26) and non-small-cell lung cancer (NSCLC) (27).

A unique feature of the IDO2 gene in humans is the high prevalence of two inactivating SNPs, which allow the opportunity to carry out loss-of-function studies directly in humans and to compare patients' data with those from $\mathrm{Ido}^{-/-}$and $\mathrm{Ido}^{+/+}$ tumor-bearing mice. These SNPs are rs10109853, which leads to a $>90 \%$ reduction in IDO2 catalytic activity (R248W), and rs4503083, which generates a premature stop codon (Y359X) and completely inactivates IDO2 activity (26). Large scale sequencing analysis revealed that these two nonfunctional alleles of IDO2 are frequently distributed in human populations of Asian, European, and African descent. However, although both of these SNPs are highly prevalent in human populations, their clinical significance has remained unclear.

\section{IDO2 in Mouse Tumors}

Similarly to human cancer, IDO2 is not frequently expressed in mouse tumors. However, the opportunity to use $I d o 2^{-/-}$mice as compared to IDO2-expressing counterparts allows the study of the function of endogenous IDO2 in tumor-bearing individuals.

Lewis lung carcinoma (LLC), isolated from a spontaneous epidermoid carcinoma of the mouse lung, does not express IDO1 and IDO2. Therefore, when injecting LLC cells into IDO2 KO mice, no IDO2 will be present anywhere in the organism. In these conditions, tumor growth is suppressed, IFN- $\gamma$ secretion is enhanced in the tumor bed, and the number of $\mathrm{CD}^{+}$tumor infiltrating lymphocytes (TILs) is increased (28).

Endogenous IDO2 may also be involved in mechanisms of tumorigenesis. To investigate this possibility, Nevler et al. (29) used the KC transgenic mouse model (30) in which an inducible oncogenic Kras allele is activated in pancreatic progenitor cells, thus leading to the development of ductal lesions that recapitulate the full spectrum of human pancreatic intraepithelial neoplasias, putative precursors to invasive pancreatic cancer (PDAC). They found that PDAC development was significantly decreased when $\mathrm{Ido}^{-/-}$alleles had been introduced into the $\mathrm{KC}$ strain via interbreeding. No major changes could be observed for immune populations infiltrating the tumor. Unexpectedly, the impact of IDO2 loss in tumor growth was mainly associated with females. In fact, no tumor development at all could be observed in $I d o 2^{-/-}$ females under study (29).

B16/BL6 melanoma is an example of mouse tumor cells that express IDO2 (31). In order to understand the role of the enzyme in this tumor, Liu et al. performed Ido2 gene silencing in vitro via small interfering RNA (siRNA). Reduction of IDO2 expression in B16/BL6 cells inhibited cancer cell proliferation, arrest of the cell cycle in G1, increased the rate of apoptosis, and reduced cell migration. These in vitro effects were accompanied by a decrease in $\mathrm{NAD}^{+}$(a metabolite downstream the kynurenine pathway). Addition of exogenous $\mathrm{NAD}^{+}$to B16/BL6 cell cultures weakened the effect of IDO2 downregulation. In vivo, B16/BL6 cells with reduced IDO2 expression grew less than IDO2-competent cells (31). The possible involvement of immune cells and of endogenous IDO2 was not addressed in this study.

Thus, as a whole, the available data would indicate that IDO2, either endogenous or expressed by the tumor, exerts immunosuppressive and pro-tumor effects in mouse models of cancer.

\section{IDO2 in Human Pancreatic Ductal Adenocarcinoma (PDAC)}

$\mathrm{PDAC}$ is one of the most aggressive and lethal diseases. Less than $10 \%$ of patients with PDAC has a life expectance of five years after diagnosis (32). Despite encouraging evidence for other tumors, including non-small-cell lung cancer (NSCLC; see below), the use of immune checkpoint inhibitors, such as antiCTLA4 (ipilimumab and tremelimumab) and anti-PD1 (nivolumab and pembrolizumab) antibodies, has shown poor efficacy in PDAC as monotherapy. Although clinical trials are undergoing with combinations of two immune checkpoint inhibitors or one immune checkpoint inhibitor and chemotherapy, the road to an effective immunotherapeutic cure for PDAC appears full of obstacles (33).

IDO2 may represent an important drug target in PDAC therapy. In fact, IDO2 is frequently upregulated in human PDAC (29). In a recent study, the analysis of the prevalence of the two IDO2-inactivating SNPs together with the treatment outcomes indicated that, in PDAC patients having received adjuvant radiotherapy, the "IDO2-deficient status" significantly associates with improved disease-free survival (29). Therefore, the IDO2 genotype has the immediate potential to influence the PDAC care decision-making process through stratification of those patients who stand to benefit from adjuvant radiotherapy.

An additional interesting aspect of IDO2 involvement in PDAC is sexual dimorphism. In fact, along the same line of Ido $2^{-/-}$female mice in which development of PDAC is significantly less than the male counterparts, female patients with PDAC rarely harbor the IDO2-deficient status (29). Therefore, these data would suggest that female patients with PDAC should be taken into high consideration for immunotherapy involving IDO2 inhibition.

\section{IDO2 in Human Non-Small Cell Lung Cancer (NSCLC)}

NSCLC, representing the majority (approximately 85\%) of lung malignancies (34), is in general insensitive to standard treatments with chemotherapeutic drugs. Therapy of NSCLC has been partly improved by the use of nivolumab. In fact, nivolumab treatment has been associated with longer overall survival than the chemotherapeutic docetaxel among patients with previously treated NSCLC, regardless of PD-L1 levels (35, 36). Nevertheless, the response rate was not more than $20 \%$. A more recent study (37) indicated that, in patients with untreated stage IV or recurrent NSCLC with a PD-L1 expression level of 5\% or more, nivolumab was not associated with longer progressionfree survival than chemotherapy. Therefore, although more 
therapeutic options are available than PDAC, alternative drug targets are also needed in NSLC.

In a recent study with 191 NSCLC patients, IDO2 was highly expressed in $84 \%$ of samples and its expression was strictly related to high PD-L1 levels (27). Perhaps most importantly, a significant correlation between IDO2 high expression and poor NSCLC prognosis was detected (27). Intriguingly, IDO2 expression was mainly associated with the basolateral side of the tumor cell membrane, and only few cells stained for IDO2 in the cytosol or nucleus. Therefore, these data would suggest a "membrane-associated" function, which may be distinct from the catalytic activity, similarly to IDO1 (12). Alternatively, the nuclear topology may further suggest a gene modulatory function by IDO2. In this regard, it is interesting to note that a previous study indicated that the nuclear-associated staining of IDO2 in the liver of conventional mice does not correlate with any difference in Trp/Kyn levels (38), thus possibly excluding the catalytic activity in nuclear-associated IDO2.

To evaluate the contribution of genetic variation in IDO2 to the risk of NSCLC, we examined the frequencies of the two common SNPs in IDO2 as described above, namely rs10109853 (R248W) and rs4503083 (Y359X). By resorting to a cohort involving 145 NSCLC patients and 395 healthy matched controls, we found that the $\mathrm{R} 248 \mathrm{~W}$ displays a significantly different genotype distribution between NSCLC patients and controls, with the genotypes that include the minor allele conferring almost a 2-fold increased risk of NSCLC (Table 1). The Y359X SNP instead displayed only a trend towards association with NSCLC and only when using a dominant genetic model. Taken together, these results highlighted genetic variation in IDO2 as a key determinant of susceptibility to NSCLC. However, the IDO2 SNPs' role appears to be distinct in NSCLC as compared to PDAC. In fact, whereas the presence of homo- or heterozygosity for the two SNPs increases the risk for NSCLC, the same condition will protect from PDAC.

Because the R248W SNP is described to impair IDO2 catalytic activity and the Y359X SNP generates a premature stop codon abolishing activity completely (26), the link observed between both SNPs and the development of NSCLC would support a relevant contribution of a defective enzymatic activity of IDO2 to disease pathogenesis. However, there are additional putative functional consequences of the IDO2 SNPs worth considering. For example, while it does not affect gene expression, R248W is described to act as a strong splicing quantitative trait locus (sQTL) of IDO2 across several tissues, but not the lung, in the Genotype-Tissue Expression (GTEx) project (Figure 1). Although the role of splicing events in IDO2 function remains unclear, the fact that the risk allele of $\mathrm{R} 248 \mathrm{~W}$ is

TABLE 1 | Association test results of IDO2 genotypes and the risk of non-small-cell lung cancer (NSCLC).

\begin{tabular}{|c|c|c|c|c|c|}
\hline Ref SNP & Genotypes & $\begin{array}{c}\text { Controls }(\mathrm{N}=395) \\
n(\%)\end{array}$ & n (\%) & $\begin{array}{c}\text { NSCLC }(\mathrm{N}=145) \\
\text { OR }(95 \% \mathrm{Cl})\end{array}$ & P-value \\
\hline & $\mathrm{T} / \mathrm{T}$ & $251(63.5)$ & 79 (54.9) & Reference & \\
\hline rs4503083 & $\mathrm{T} / \mathrm{A}$ & $131(33.2)$ & 56 (38.9) & $1.36(0.91-2.03)$ & 0.145 \\
\hline \multirow[t]{3}{*}{ (Y359X) } & AVA & $13(3.3)$ & 9 (6.3) & $2.20(0.91-5.34)$ & 0.123 \\
\hline & $\mathrm{T} / \mathrm{A}+\mathrm{A} / \mathrm{A}$ & $144(36.5)$ & $65(45.1)$ & $1.43(0.97-2.11)$ & 0.073 \\
\hline & $\mathrm{T} / \mathrm{T}$ & 118 (29.9) & 28 (19.3) & Reference & \\
\hline rs10109853 & $\mathrm{T} / \mathrm{C}$ & $192(48.6)$ & $88(60.7)$ & $1.93(1.19-3.13)$ & 0.008 \\
\hline \multirow[t]{2}{*}{ (R248W) } & $\mathrm{C} / \mathrm{C}$ & $85(21.5)$ & $29(20.0)$ & $1.44(0.80-2.59)$ & 0.231 \\
\hline & $\mathrm{T} / \mathrm{C}+\mathrm{C} / \mathrm{C}$ & $277(70.1)$ & $117(80.9)$ & $1.78(1.12-2.84)$ & 0.016 \\
\hline
\end{tabular}

SNP, single nucleotide polymorphism; NSCLC, non-small-cell lung cancer; OR, odds ratio. One out of the 145 NSCLC patients had a missing genotype for rs4503083. Significant values are in bold.

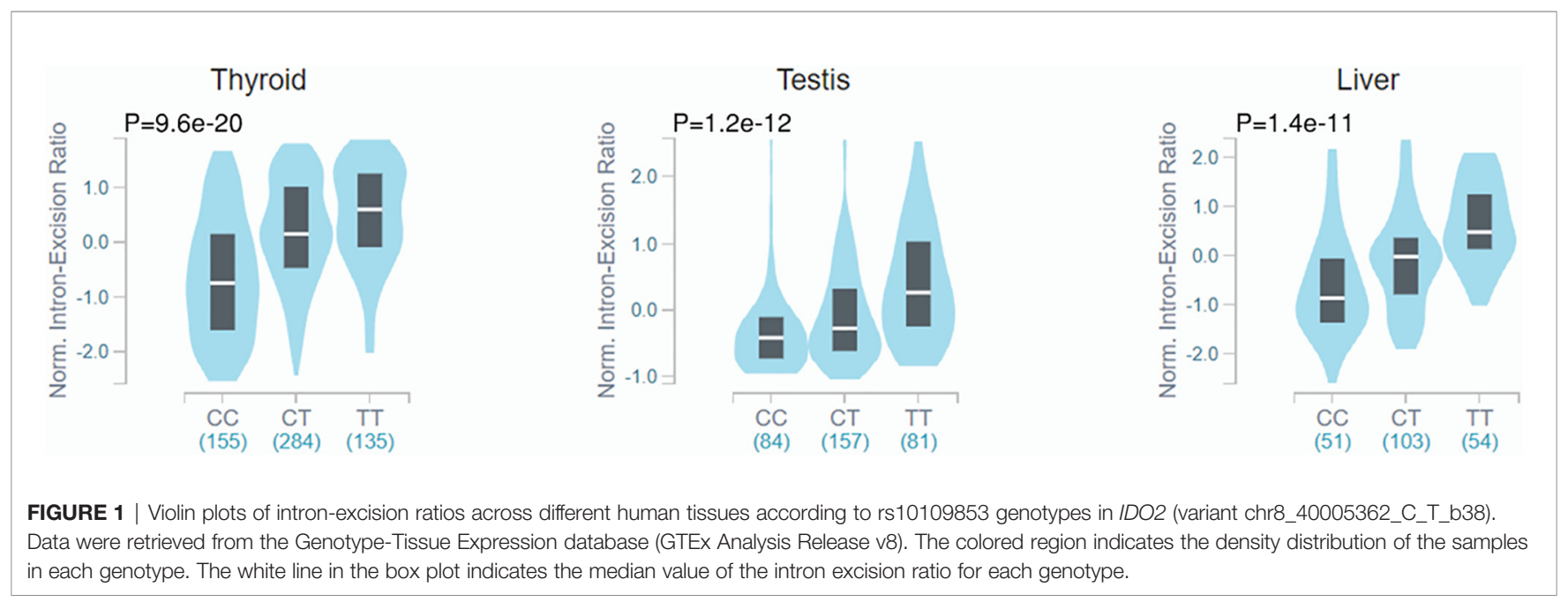

Frontiers in Immunology | www.frontiersin.org 
TABLE 2 | Main structural and functional features of human IDO1 and IDO2.

\begin{tabular}{|c|c|c|c|c|}
\hline & Features & hID01 & hID02 & References \\
\hline \multirow[t]{6}{*}{ Structural } & Trp metabolizing activity & $\mathrm{K}_{\text {cat }}\left(\mathrm{s}^{-1}\right) 2.97 \pm 0.20$ & $K_{\text {cat }}\left(s^{-1}\right) 0.103 \pm 0.006$ & $(17)$ \\
\hline & & $\mathrm{K}_{\mathrm{m}}(\mu \mathrm{M}) 20.90 \pm 3.95$ & $\mathrm{~K}_{\mathrm{m}}(\mu \mathrm{M}) 6,809 \pm 917$ & \\
\hline & Presence of signaling motifs & VPYCQL (ITIM1) Signaling activity & Absent & $(10,11)$ \\
\hline & and their function & VYEGF (ITIM2) Signaling activity, protein & MYEGV (putative ITIM) & \\
\hline & & degradation & Unknown & \\
\hline & & YENM PI3K binding & Absent & $(12)$ \\
\hline \multirow[t]{3}{*}{ Functional } & $\begin{array}{l}\text { Frequency of expression in } \\
\text { tumors }\end{array}$ & High & Low & $(13,24,26,27)$ \\
\hline & Type of tumors & $\begin{array}{l}\text { Endometrial, cervical, renal, gastric, and } \\
\text { colorectal carcimonas Glioblastoma }\end{array}$ & $\begin{array}{l}\text { Renal, gastric, and colorectal carcimonas Pancreas } \\
\text { (PDAC), lung (NSCLC carcinomas) }\end{array}$ & $(13,24,26,27)$ \\
\hline & Function in tumors & Immune escape & Not well defined, may be dependent on the genotype & $(9,14,29)$ \\
\hline
\end{tabular}

reported to influence the intron-excision ratio of $I D O 2$ suggests an effect on transcript diversity that may also help explain its stronger association with NSCLC.

\section{CONCLUSION AND PERSPECTIVES}

IDO2 is a protein molecule that may represent an important drug target in cancer immunotherapy. In fact, IDO2 has some Trp catabolic activity, a catalytic function that, in the case of IDO1, has been demonstrated to be responsible for immunoregulatory effects. However, IDO2 is a very poor producer of Kyn and, consequently, Trp catabolic activity by this enzyme can very unlikely account for IDO2 biologic effects. In this regard, IDO2 may represent a sort of "pseudoenzyme", i.e., a protein that is evolutionarily related to active enzymes, but lacks relevant catalytic activity (Table 2) $(39,40)$. Interestingly, the biological meaning of pseudoenzymes is currently the focus of intense research (41). Some authors suggested that the IDO2 catalytic function may depend on factors (42) whose identity has not been entirely identified yet and, therefore, it may have a better performance in "certain" in vivo conditions. However, as this is a hypothesis, the use of IDO2 catalytic inhibitors would be premature in cancer immunotherapy. An alternative hypothesis could in fact be that IDO2 preferentially uses substrates other than Trp and therefore a completely new story should be written for IDO2 as an enzyme. Disappointingly, no evidence for an alternative IDO2 catalytic activity has been provided so far. A great help in this regard may come from the crystallization of the IDO2 protein, which, unfortunately, has not been obtained yet.

The fact that IDO2 biology is still far from being understood also derives from the observations that this molecule appears to play opposite functions in both autoimmune and neoplastic diseases. In fact, in mouse experimental models of autoimmunity/chronic inflammation, IDO2 is pathogenetic in arthritis $(20,43)$ and protective in psoriasis (22). In humans, the presence of an IDO2-deficient functional status exerts protective effects in PDAC (29) but increases the risk of developing NSCLC (this study). As a whole, these data would suggest that IDO2 plays a context-dependent effect. In other words, the presence of cell- or microenvironment-specific factors as well as the direct interaction with specific protein partners would dictate the outcome of IDO2-associated effects. In this regard, the determination of the IDO2 interactome may be of great help. Unfortunately, at this time, we just know which known IDO1 partners do not interact with IDO2 (Table 2). These include SHP-1 and SHP-2 phosphatases that interact with IDO1 ITIM1 (mediating immunoregulatory IDO1 signaling activity in DCs (10); i.e., absent in IDO2) and class I PI3Ks that bind IDO1 via the YENM motif (mediating early endosome localization and thus the signaling function of IDO1 in DCs and tumor cell transfectants (12); also absent in IDO2). The ITIM2 motif is instead present in both mouse and human IDO2, but its role is still unknown. Interestingly, a recent study indicated that, upon treatment with lipopolysaccharide (LPS), IDO2 exerts negative regulatory effects on the IL- 6 signaling pathway by reducing STAT3 expression in macrophages and possibly in other cell types in vivo (44). Notably, this effect occurred without changes in Kyn levels (44) and therefore it could be of much interest to clarify the molecular mode of action of IDO2 in this context.

In human neoplasia, although studies in only two types of tumor have been performed, a relevant issue appears to be the IDO2 genotype, whose analysis could provide a valuable biomarker for informing treatment decisions (29). However, even in conditions such as PDAC in which the IDO2 loss-offunction seems to be protective, the fact that we do not know the true function/s of IDO2 in distinct cells and cellular microenvironments may have important consequences. As just an example, the use of IDO2 catalytic inhibitors may induce effects also in immune cells that should mount an effective antitumor response in neoplastic patients. Moreover, given the lack of information of a validated function, allocation of distinct profiles of IDO2 expression to the identified molecular subtypes of PDAC (45) cannot be performed yet.

In conclusion, although compounds that simultaneously inhibit IDO1 and IDO2 have already been identified (46), we believe that there is still a long road ahead before drug targeting of IDO2 can be effectively and safely used in cancer immunotherapy.

\section{DATA AVAILABILITY STATEMENT}

The raw data supporting the conclusions of this article will be made available by the authors, without undue reservation. 


\section{ETHICS STATEMENT}

The studies involving human participants were reviewed and approved by Comitato Etico Regionale dell'Umbria. The patients/participants provided their written informed consent to participate in this study.

\section{AUTHOR CONTRIBUTIONS}

All authors contributed to the article and approved the submitted version.

\section{REFERENCES}

1. Murray PJ. Amino Acid Auxotrophy as a System of Immunological Control Nodes. Nat Immunol (2016) 17(2):132-9. doi: 10.1038/ni.3323

2. Grohmann U, Bronte V. Control of Immune Response by Amino Acid Metabolism. Immunol Rev (2010) 236:243-64. doi: 10.1111/j.1600-065X. 2010.00915.x

3. Mondanelli G, Mondanelli G, Ugel S, Grohmann U, Bronte V. The Immune Regulation in Cancer by the Amino Acid Metabolizing Enzymes ARG and IDO. Curr Opin Pharmacol (2017) 35:30-9. doi: 10.1016/j.coph.2017.05.002

4. Nguyen NT, Kimura A, Nakahama T, Chinen I, Masuda K, Nohara K, et al. Aryl Hydrocarbon Receptor Negatively Regulates Dendritic Cell Immunogenicity Via a Kynurenine-Dependent Mechanism. Proc Natl Acad Sci USA (2010) 107(46):19961-6. doi: 10.1073/pnas.1014465107

5. Bessede A, Gargaro M, Pallotta MT, Matino D, Servillo G, Brunacci C, et al. Aryl Hydrocarbon Receptor Control of a Disease Tolerance Defence Pathway. Nature (2014) 511(7508):184-90. doi: 10.1038/nature13323

6. Rothhammer V, Quintana FJ. The Aryl Hydrocarbon Receptor: An Environmental Sensor Integrating Immune Responses in Health and Disease. Nat Rev Immunol (2019) 19(3):184-97. doi: 10.1038/s41577-019-0125-8

7. Mezrich JD, Fechner JH, Zhang X, Johnson BP, Burlingham WJ, Bradfield CA. An Interaction Between Kynurenine and the Aryl Hydrocarbon Receptor Can Generate Regulatory T Cells. J Immunol (2010) 185(6):3190-8. doi: 10.4049/jimmunol.0903670

8. Mellor AL, Munn DH. IDO Expression by Dendritic Cells: Tolerance and Tryptophan Catabolism. Nat Rev Immunol (2004) 4(10):762-74. doi: 10.1038/ nri1457

9. Prendergast GC, Malachowski WP, DuHadaway JB, Muller AJ. Discovery of IDO1 Inhibitors: From Bench to Bedside. Cancer Res (2017) 77(24):6795-811. doi: 10.1158/0008-5472.CAN-17-2285

10. Pallotta MT, Orabona C, Volpi C, Vacca C, Belladonna ML, Bianchi R, et al. Indoleamine 2,3-Dioxygenase is a Signaling Protein in Long-Term Tolerance by Dendritic Cells. Nat Immunol (2011) 12(9):870-8. doi: 10.1038/ni.2077

11. Albini E, Rosini V, Gargaro M, Mondanelli G, Belladonna ML, Pallotta MT, et al. Distinct Roles of Immunoreceptor Tyrosine-Based Motifs in Immunosuppressive Indoleamine 2,3-Dioxygenase 1. J Cell Mol Med (2017) 21(1):165-76. doi: 10.1111/jcmm.12954

12. Iacono A, Pompa A, De Marchis F, Panfili E, Greco FA, Coletti A, et al. Class IA PI3Ks Regulate Subcellular and Functional Dynamics of IDO1. EMBO Rep (2020) 21(12):e49756. doi: 10.15252/embr.201949756

13. van Baren N, Van den Eynde BJ. Tryptophan-Degrading Enzymes in Tumoral Immune Resistance. Front Immunol (2015) 6:34. doi: 10.3389/fimmu. 2015.00034

14. Vacchelli E, Aranda F, Eggermont A, Sautes-Fridman C, Tartour E, Kennedy EP, et al. Trial Watch: IDO Inhibitors in Cancer Therapy. Oncoimmunology (2014) 3(10):e957994. doi: 10.4161/21624011.2014.957994

15. Long GV, Dummer R, Hamid D, Gajewski TF, Caglevic C, Dalle S, et al. Epacadostat Plus Pembrolizumab Versus Placebo Plus Pembrolizumab in Patients With Unresectable or Metastatic Melanoma (ECHO-301/ KEYNOTE-252): A Phase 3, Randomised, Double-Blind Study. Lancet Oncol (2019) 20(8):1083-97. doi: 10.1016/S1470-2045(19)30274-8

\section{FUNDING}

This work was supported by Associazione Italiana per la Ricerca sul Cancro (AIRC 2019-23084; to UG) and the Italian Ministry of Education, University, and Research (PRIN 20173EAZ2Z; to CV). AC was supported by the Fundação para a Ciência e a Tecnologia (FCT) (UIDB/50026/2020, UIDP/50026/2020, CEECIND/03628/2017 and PTDC/MED-GEN/28778/2017) and the Northern Portugal Regional Operational Programme (NORTE 2020), under the Portugal 2020 Partnership Agreement, through the European Regional Development Fund (ERDF) (NORTE-01-0145-FEDER-000039).

16. Ball HJ, Sanchez-Perez A, Weiser S, Austin CJ, Astelbauer F, Miu J, et al. Characterization of an Indoleamine 2,3-Dioxygenase-Like Protein Found in Humans and Mice. Gene (2007) 396(1):203-13. doi: 10.1016/j.gene.2007. 04.010

17. Pantouris G, Serys M, Yuasa HJ, Ball HJ, Mowat CG. Human Indoleamine 2,3-Dioxygenase-2 Has Substrate Specificity and Inhibition Characteristics Distinct From Those of Indoleamine 2,3-Dioxygenase-1. Amino Acids (2014) 46(9):2155-63. doi: 10.1007/s00726-014-1766-3

18. Zhai L, Bell A, Ladomersky E, Lauing KL, Bollu L, Sosman JA, et al. Immunosuppressive IDO in Cancer: Mechanisms of Action, Animal Models, and Targeting Strategies. Front Immunol (2020) 11:1185. doi: 10.3389/fimmu.2020.01185

19. Metz R, Smith C, DuHadaway JB, Chandler P, Baban B, Merlo LM, et al. IDO2 is Critical for IDO1-Mediated T-Cell Regulation and Exerts a Non-Redundant Function in Inflammation. Int Immunol (2014) 26(7):357-67. doi: 10.1093/ intimm/dxt073

20. Merlo LMF, Pigott E, DuHadaway JB, Grabler S, Metz R, Prendergast GC, Mandik-Nayak L, et al. IDO2 is a Critical Mediator of Autoantibody Production and Inflammatory Pathogenesis in a Mouse Model of Autoimmune Arthritis. J Immunol (2014) 192(5):2082-90. doi: 10.4049/ jimmunol.1303012

21. Merlo LM, DuHadaway JB, Grabler S, Prendergast GC, Muller AJ, MandikNayak L. IDO2 Modulates T Cell-Dependent Autoimmune Responses Through a B Cell-Intrinsic Mechanism. J Immunol (2016) 196(11):4487-97. doi: 10.4049/jimmunol.1600141

22. Fujii K, Yamamoto Y, Mizutani Y, Saito K, Seishima M. Indoleamine 2,3Dioxygenase 2 Deficiency Exacerbates Imiquimod-Induced Psoriasis-Like Skin Inflammation. Int J Mol Sci (2020) 21:(15). doi: 10.3390/ijms21155515

23. Napolioni V, Pariano M, Borghi M, Oikonomou V, Galosi C, De Luca A, et al. Genetic Polymorphisms Affecting IDO1 or IDO2 Activity Differently Associate With Aspergillosis in Humans. Front Immunol (2019) 10:890. doi: 10.3389/fimmu.2019.00890

24. Lob S, Konigsrainer A, Schafer R, Rammensee HG, Opelz G, Terness P. LevoBut Not Dextro-1-Methyl Tryptophan Abrogates the IDO Activity of Human Dendritic Cells. Blood (2008) 111(4):2152-4. doi: 10.1182/blood-2007-10116111

25. Guastella AR, Michelhaugh SK, Klinger NV, Fadel HA, Kiousis S, Ali-Fehmi $\mathrm{R}$, et al. Investigation of the Aryl Hydrocarbon Receptor and the Intrinsic Tumoral Component of the Kynurenine Pathway of Tryptophan Metabolism in Primary Brain Tumors. J Neurooncol (2018) 139(2):239-49. doi: 10.1007/ s11060-018-2869-6

26. Witkiewicz AK, Costantino CL, Metz R, Muller AJ, Prendergast GC, Yeo CJ, et al. Genotyping and Expression Analysis of IDO2 in Human Pancreatic Cancer: A Novel, Active Target. J Am Coll Surg (2009) 208(5):781-7; discussion 787-9. doi: 10.1016/j.jamcollsurg.2008.12.018

27. Mandarano M, Bellezza G, Belladonna ML, Vannucci J, Gili A, Ferri I, et al. Indoleamine 2,3-Dioxygenase 2 Immunohistochemical Expression in Resected Human Non-Small Cell Lung Cancer: A Potential New Prognostic Tool. Front Immunol (2020) 11:839. doi: 10.3389/fimmu.2020.00839

28. Yamasuge W, Yamamoto Y, Fujigaki H, Hoshi M, Nakamoto K, Kunisawa K, et al. Indoleamine 2,3-Dioxygenase 2 Depletion Suppresses Tumor Growth in 
a Mouse Model of Lewis Lung Carcinoma. Cancer Sci (2019) 110(10):3061-7. doi: 10.1111/cas.14179

29. Nevler A, Muller AJ, Sutanto-Ward E, DuHadaway JB, Nagatomo K, Londin E, et al. Host IDO2 Gene Status Influences Tumor Progression and Radiotherapy Response in KRAS-Driven Sporadic Pancreatic Cancers. Clin Cancer Res (2019) 25(2):724-34. doi: 10.1158/1078-0432.CCR-18-0814

30. Hingorani SR, Petricoin EF, Maitra A, Rajapakse V, King C, Jacobetz MA, et al. Preinvasive and Invasive Ductal Pancreatic Cancer and Its Early Detection in the Mouse. Cancer Cell (2003) 4(6):437-50. doi: 10.1016/ S1535-6108(03)00309-X

31. Liu Y, Zhang Y, Zheng X, Zhang X, Wang H, Li Q, et al. Gene Silencing of Indoleamine 2,3-Dioxygenase 2 in Melanoma Cells Induces Apoptosis Through the Suppression of NAD+ and Inhibits in Vivo Tumor Growth. Oncotarget (2016) 7(22):32329-40. doi: 10.18632/oncotarget.8617

32. Miller KD, Siegel RL, Lin CC, Mariotto AB, Kramer JL, Rowland JH, et al. Cancer Treatment and Survivorship Statistics, 2016. CA Cancer J Clin (2016) 66(4):271-89. doi:10.3322/caac.21349

33. Sarantis P, Koustas E, Papadimitropoulou A, Papavassiliou AG, Karamouzis MV. Pancreatic Ductal Adenocarcinoma: Treatment Hurdles, Tumor Microenvironment and Immunotherapy. World J Gastrointest Oncol (2020) 12(2):173-81. doi: 10.4251/wjgo.v12.i2.173

34. Travis WD, Brambilla E, Noguchi M, Nicholson AG, Geisinger KR, Yatabe Y, et al. International Association for the Study of Lung Cancer/American Thoracic Society/European Respiratory Society International Multidisciplinary Classification of Lung Adenocarcinoma. J Thorac Oncol (2011) 6(2):244-85. doi: 10.1097/JTO.0b013e318206a221

35. Brahmer J, Reckamp KL, Baas P, Crino L, Eberhardt WE, Poddubskaya E, et al. Nivolumab Versus Docetaxel in Advanced Squamous-Cell Non-Small-Cell Lung Cancer. N Engl J Med (2015) 373(2):123-35. doi: 10.1056/NEJMoa1504627

36. Borghaei H, Paz-Ares L, Horn L, Spigel DR, Steins M, Ready NE, et al. Nivolumab Versus Docetaxel in Advanced Nonsquamous Non-Small-Cell Lung Cancer. N Engl J Med (2015) 373(17):1627-39. doi: 10.1056/ NEJMoa1507643

37. Carbone DP, Reck M, Paz-Ares L, Creelan B, Horn L, Steins M, et al. FirstLine Nivolumab in Stage IV or Recurrent Non-Small-Cell Lung Cancer. N Engl J Med (2017) 376(25):2415-26. doi: 10.1056/NEJMoa1613493

38. Jusof FF, Bakmiwewa SM, Weiser S, Too LK, Metz R, Prendergast GC, et al. Investigation of the Tissue Distribution and Physiological Roles of Indoleamine 2,3-Dioxygenase-2. Int J Tryptophan Res (2017) 10:1178646917735098. doi: 10.1177/1178646917735098
39. Wang TY, Zhao J, Savas AC, Zhang S, Feng P. Viral Pseudoenzymes in Infection and Immunity. FEBS $J$ (2020) 287(19):4300-9. doi: 10.1111/febs. 15545

40. Ribeiro AJM, Das S, Dawson N, Zaru S, Thornton JM, et al. Emerging Concepts in Pseudoenzyme Classification, Evolution, and Signaling. Sci Signal (2019) 12:(594). doi: 10.1126/scisignal.aat9797

41. Murphy JM, Mace PD, Eyers PA. Live and Let Die: Insights Into Pseudoenzyme Mechanisms From Structure. Curr Opin Struct Biol (2017) 47:95-104. doi: 10.1016/j.sbi.2017.07.004

42. Austin CJ, Mailu BM, Maghzal GJ, Sanchez-Perez A, Rahlfs S, Zocher K, et al. Biochemical Characteristics and Inhibitor Selectivity of Mouse Indoleamine 2,3-Dioxygenase-2. Amino Acids (2010) 39(2):565-78. doi: 10.1007/s00726010-0475-9

43. Prendergast GC, Metz R, Muller AJ, Merlo LM, Mandik-Nayak L. IDO2 in Immunomodulation and Autoimmune Disease. Front Immunol (2014) 5:585. doi: 10.3389/fimmu.2014.00585

44. Yamamoto Y, Yamasuge W, Imai S, Kunisawa K, Hoshi M, Fujigaki H, et al. Lipopolysaccharide Shock Reveals the Immune Function of Indoleamine 2,3Dioxygenase 2 Through the Regulation of IL-6/Stat3 Signalling. Sci Rep (2018) 8(1):15917. doi: 10.1038/s41598-018-34166-4

45. Bailey P, Chang DK, Nones K, Johns AL, Patch AM, Gingras MC, et al. Genomic Analyses Identify Molecular Subtypes of Pancreatic Cancer. Nature (2016) 531(7592):47-52.

46. Winters M, DuHadaway JB, Pham KN, Lewis-Ballester A, Badir S, Wai J, et al. Diaryl Hydroxylamines as Pan or Dual Inhibitors of Indoleamine 2,3Dioxygenase-1, Indoleamine 2,3-Dioxygenase-2 and Tryptophan Dioxygenase. Eur J Med Chem (2019) 162:455-64. doi: 10.1016/j.ejmech. 2018.11.010

Conflict of Interest: The authors declare that the research was conducted in the absence of any commercial or financial relationships that could be construed as a potential conflict of interest.

Copyright (๑) 2021 Mondanelli, Mandarano, Belladonna, Suvieri, Pelliccia, Bellezza, Sidoni, Carvalho, Grohmann and Volpi. This is an open-access article distributed under the terms of the Creative Commons Attribution License (CC BY). The use, distribution or reproduction in other forums is permitted, provided the original author(s) and the copyright owner(s) are credited and that the original publication in this journal is cited, in accordance with accepted academic practice. No use, distribution or reproduction is permitted which does not comply with these terms. 